\title{
Reply to Ghirardello et al Letter to the Editor
}

\author{
Rozeta Sokou ${ }^{1}$ Daniele Piovani ${ }^{2,3}$ Aikaterini Konstantinidi ${ }^{1}$ Andreas G. Tsantes ${ }^{4}$ \\ Stavroula Parastatidou ${ }^{1}$ Maria Lampridou ${ }^{1}$ Georgios loakeimidis ${ }^{1}$ Antonis Gounaris ${ }^{5}$ \\ Nicoletta lacovidou ${ }^{6}$ Anastasios G. Kriebardis ${ }^{7}$ Marianna Politou ${ }^{8}$ Petros Kopterides ${ }^{9}$ \\ Stefanos Bonovas ${ }^{2,3}$ Argirios E. Tsantes ${ }^{4}$
}

${ }^{1}$ Neonatal Intensive Care Unit, "Agios Panteleimon" General Hospital of Nikea, Piraeus, Greece

${ }^{2}$ Department of Biomedical Sciences, Humanitas University, Milan, Italy

${ }^{3}$ Humanitas Clinical and Research Center-IRCCS, Milan, Italy

4 Laboratory of Haematology and Blood Bank Unit, "Attiko" Hospital, School of Medicine, National and Kapodistrian University of Athens, Athens, Greece

${ }^{5}$ Neonatal Intensive Care Unit, University Hospital of Larissa, Larissa, Greece

${ }^{6}$ Neonatal Department, Aretaeio Hospital, National and Kapodistrian University of Athens, Athens, Greece

${ }^{7}$ Laboratory of Reliability and Quality Control in Laboratory Hematology, Department of Biomedical Science, School of Health and Caring Science, University of West Attica, Egaleo, Greece

8 Department of Blood Transfusion, Aretaieion Hospital, School of Medicine, National and Kapodistrian University of Athens, Athens, Greece

9 Intensive Care Unit, Excela Health Westmoreland Hospital, Greensburg, Pennsylvania, United States

Thromb Haemost 2021;121:1119-1120.

We thank Dr. Ghirardello and colleagues ${ }^{1}$ for their interest in our study, which introduced an easy-to-apply, rotational thromboelastometry-based, prediction model of hemorrhage in critically ill neonates. ${ }^{2}$ Their letter gives us the opportunity to address their comments and provide clarifications to their assumptions.

First, regarding our previous work "Thromboelastometry variables in neonates with perinatal hypoxia," ${ }^{3}$ Ghirardello et al note that "no cases of intraventricular hemorrhage (IVH) were recorded." Actually, $41 \mathrm{IVH}$ events of any grade were recorded in this population, but they were not reported in this particular paper. $^{3}$ These data are fully reported in the $\mathrm{PhD}$ thesis. ${ }^{4}$ On the other hand, it is true that "we did not apply the NeoBAT score to assess bleeding in this study"; nevertheless, Ghirardello et al rather lose sight of the fact that the aim of this study ${ }^{3}$ was to investigate the hemostatic profile of neonates with perinatal hypoxia, and not to assess clinical outcomes: there was no reason to use the NeoBAT score for bleeding assessment in this study. ${ }^{3}$

Second, in our recent publication regarding the development of the Neonatal Bleeding Risk (NeoBRis) index, ${ }^{2}$ the
Address for correspondence Argirios E. Tsantes, MD, PhD, Laboratory of Haematology and Blood Bank Unit, "Attiko" Hospital, School of Medicine, National and Kapodistrian University of Athens, 1 Rimini Str., 12462 Athens, Greece (e-mail: atsantes@yahoo.com).

incidence of IVH was approximately $29 \%$ across the entire population of critically ill neonates with gestational ages between 24 and 42 weeks (not between 32 and 39 weeks, as stated by Ghirardello et al; Table 1 of our original manuscript $^{2}$ clearly reports the interquartile range). In fact, 72 (21.7\%) neonates had gestational age $\leq 30$ weeks, 89 (26.8\%) had a birth weight $<1,500 \mathrm{~g}$, and the IVH events included hemorrhages of any grade, not only severe IVH.

Third, Ghirardello et al point out that large observational studies have determined the incidence of IVH at approximately $1 \%$ in neonates over 30 weeks of gestational age, and to support this claim they cite three studies. ${ }^{5-7}$ However, they do not mention that Harding et al reported a risk of $1.1 \%$ for IVH of grade 2 or higher, while among 364 babies born before 30 weeks, the IVH incidence was $22 \% .^{5}$ Bhat et al detected an incidence of IVH ranging from 3.3 to $6.3 \%$ at various gestational ages (over 30 weeks). ${ }^{6}$ Finally, Ballardini et al studied all latepreterm infants, not only those being critically ill. ${ }^{7}$ It is well known that several risk factors, including sepsis, development of respiratory distress syndrome, and hypoxia predispose to IVH. $^{8}$ Taking into consideration that (1) our study population published online

December 9, 2020 (c) 2020. Thieme. All rights reserved. Georg Thieme Verlag KG,

Rüdigerstraße 14,

70469 Stuttgart, Germany
DOI https://doi.org/ 10.1055/a-1333-7387. ISSN 0340-6245. 
consisted of critically ill neonates with gestational age of 24 to 42 weeks and (2) we analyzed IVH events of any grade, ${ }^{2}$ our findings are not fully comparable with those of the studies ${ }^{5-7}$ cited by Ghirardello et al. In addition, the incidence of IVH (i.e., $29 \%$ ) detected in our study is in line with previous studies conducted in preterm infants (24-31\%), ${ }^{9,10}$ while relevant data for critically ill neonates are scarce.

Regarding the comment that we "did not provide clinical data to better characterize the severity of illness for patients enrolled" in our work, the study design and the inclusion criteria have been clearly reported in the Methods section. ${ }^{2}$ We will be happy to provide specific additional clinical data if available in our dataset.

We invite Ghirardello and colleagues to validate our prognostic index of hemorrhage in critically ill neonates. ${ }^{2}$ As with any novel prognostic index, it needs to be improved by external prospective studies, to account for potential differences in health care systems, measurement methods, and patient characteristics.

Funding

None.

Conflict of Interest

None declared.

\section{References}

1 Ghirardello S, Raffaeli G, Mosca F. In reply to: A risk score for predicting the incidence of hemorrhage in critically ill neonates: development and validation study. Thromb Haemost 2020. Doi: 10.1055/s-0040-1721316

2 Sokou R, Piovani D, Konstantinidi A, et al. A risk score for predicting the incidence of hemorrhage in critically ill neonates: development and validation study. Thromb Haemost 121(2): 131-139

3 Konstantinidi A, Sokou R, Tsantes AG, et al. Thromboelastometry variables in neonates with perinatal hypoxia. Semin Thromb Hemost 2020;46(04):428-434

4 Konstantinidi A. Alterations of Thromboelastographic Variables in Neonates with Perinatal Hypoxia/Asphyxia and Potential Use of This Method as Prognostic and Therapeutic Biomarker for These Neonates [Ph.D. dissertation]. Athens, Greece: National and Kapodistrian University of Athens; 2020. Accessed January 5, 2021 at: https://www.didaktorika.gr/eadd/handle/10442/47223

5 Harding D, Kuschel C, Evans N. Should preterm infants born after 29 weeks' gestation be screened for intraventricular haemorrhage? J Paediatr Child Health 1998;34(01):57-59

6 Bhat V, Karam M, Saslow J, et al. Utility of performing routine head ultrasounds in preterm infants with gestational age 30-34 weeks. J Matern Fetal Neonatal Med 2012;25(02):116-119

7 Ballardini E, Tarocco A, Baldan A, Antoniazzi E, Garani G, BorgnaPignatti $C$. Universal cranial ultrasound screening in preterm infants with gestational age 33-36 weeks. A retrospective analysis of 724 newborns. Pediatr Neurol 2014;51(06):790-794

8 Ballabh P. Pathogenesis and prevention of intraventricular hemorrhage. Clin Perinatol 2014;41(01):47-67

9 Lu H, Wang Q, Lu J, Zhang Q, Kumar P. Risk factors for intraventricular hemorrhage in preterm infants born at 34 weeks of gestation or less following preterm premature rupture of membranes. J Stroke Cerebrovasc Dis 2016;25(04):807-812

10 Radic JA, Vincer M, McNeely PD. Temporal trends of intraventricular hemorrhage of prematurity in Nova Scotia from 1993 to 2012. J Neurosurg Pediatr 2015;15(06):573-579 